\title{
A VARIATIONAL CALCULATION OF NUCLEAR MATTER BINDING WITH THE REID POTENTIAL
}

\author{
J.C. OWEN \\ Department of Theoretical Physics, Manchester University, Manchester M13 9PL, UK \\ and \\ R.F. BISHOP and J.M. IRVINE \\ Department of Theoretical Physics, Manchester University, Manchester M13 9PL, UK \\ and Science Research Council, Daresbury Laboratory, Daresbury, Warrington WA4 4AD, UK
}

\section{Received 21 October 1975}

\begin{abstract}
A constrained variational calculation for the binding energy of nuclear matter using the Reid soft core interaction and with tensor correlations treated exactly, is presented. Saturation is achieved at a density given by $k_{\mathrm{F}} \approx 1.75 \mathrm{fm}^{-1}$ with a binding energy of $22 \mathrm{MeV}$ per nucleon. This represents considerably more binding than is obtained in low order Brueckner perturbation calculations or in Pandharipande's lowest order constrained variational approach.
\end{abstract}

In earlier papers $[1,2]$ we have proposed an alternative to the Pandharipande approach $[3,4]$ to the calculation of the binding energy of many-body systems. Our approach can be justified on strict variational grounds (in contrast to that of Pandharipande), and gives excellent agreement with the essentially exact results [5] for the "homework problem" of Bethe over a wide range of densities. At high densities the results obtained using Pandharipande's lowest order constrained variational (LOCV) prescription diverge greatly from both our variational results and the exact results. In calculations on neutron matter using a modified central form of the Reid soft core potential [6] suggested by Pandharipande [3], we again observed discrepancies between the results of our variational calculations and the LOCV calculations of Pandharipande at high densities.

We have now considered Fermi systems containing strong two-body tensor forces capable of inducing noncentral two-body correlations. A full account of these calculations will be published elsewhere. In this letter we wish to report the results of our calculations on nuclear matter using the exact Reid soft core potential [6]. Interest in this problem has been greatly renewed recently by Pandharipande's suggestion [7] that neither LOCV nor low order Brueckner theory (LOBT) calculations adequately describe nuclear matter, and by his claim that the Reid potential yields $\gtrsim 23 \mathrm{MeV}$ per nucleon binding at $k_{\mathrm{F}}=1.6 \mathrm{fm}^{-1}$, in contrast to the value $\approx 11 \mathrm{MeV}$ per nucleon with $k_{\mathrm{F}}=1.4 \mathrm{fm}^{-1}$ at saturation for LOBT calculations and his similar LOCV results.

We consider potentials of the Reid form,

$V=\sum_{\alpha} V_{\alpha} P_{\alpha}$

where $P_{\alpha} \equiv|\alpha\rangle\langle\alpha|$ is the $\alpha$-channel projection operator, $\alpha \equiv(J S T)$. The potential $V_{\alpha}$ in general may contain central, tensor and spin-orbit components,

$V_{\alpha}=V_{\alpha}^{\mathrm{C}}+V_{\alpha}^{\mathrm{T}} S_{12}+V_{\alpha}^{\mathrm{LS}} \boldsymbol{L} \cdot \boldsymbol{S}$,

with the usual tensor operator,

$S_{12}(\hat{r})=3\left(\sigma_{1} \cdot \hat{r}\right)\left(\sigma_{2} \cdot \hat{r}\right)-\sigma_{1} \cdot \sigma_{2}$.

The corresponding generalization of the two-body correlation function that we assume is,

$f(12)=\sum_{\alpha} f_{\alpha}(12) P_{\alpha}$.

For those channels with no tensor interaction $\left(V_{\alpha}^{\mathrm{T}}\right.$ $=0$ ), we assume

$f_{\alpha}(12)=f_{\alpha}^{(1)}\left(r_{12}\right)$,

and for channels containing a tensor interaction, we explicitly allow for tensor correlations, 
$f_{\alpha}(12)=f_{\alpha}^{\mathrm{C}}\left(r_{12}\right)+f_{\alpha}^{\mathrm{T}}\left(r_{12}\right) S_{12}\left(\hat{r}_{12}\right)$.

It is most convenient [8] in these tensor-correlated channels to rewrite this expression in terms of two new mutually or thogonal projection operators, $Q$ and $\bar{Q} \equiv 1-Q$, with $Q^{2}=Q$,

$f_{\alpha}(12)=f_{\alpha}^{(2)}\left(r_{12}\right) Q\left(\hat{r}_{12}\right)+f_{\alpha}^{(3)}\left(r_{12}\right) \bar{Q}\left(\hat{r}_{12}\right)$.

Since $S_{12}^{2}=4 S(S+1)-2 S_{12}$, and since the tensor interaction acts only in spin-triplet states, it is readily shown that

$Q=\frac{1}{6}\left(S_{12}+4\right)$.

It should be noted that by writing the correlation function in the form of eqs. (4)-(6), we have explicitly correlated the tensor force but not the spin-orbit splittings. As usual the correlation function $f(12)$ is required to tend to unity as $r_{12} \rightarrow \infty$, and the orthogonality properties of the projectors $P_{\alpha}$ and the operators $Q$ and $\bar{Q}$ then imply that $f_{\alpha}^{(k)}\left(r_{12}\right) \rightarrow 1$ as $r_{12} \rightarrow \infty$ for each value of $\alpha$ and $k(=1$ or 2,3$)$ separately.

Our calculation now proceeds as before $[1,2]$. The two-body cluster energy, $E_{2}$,

$$
\begin{aligned}
& E_{2} / N=\sum_{i<j}\langle i j|-\frac{1}{2} \hbar^{2} m^{-1}\left[f(r),\left[\nabla^{2}, f(r)\right]\right] \\
& \quad+f(r) V(r) f(r)|i j-j i\rangle,
\end{aligned}
$$

is minimized with respect to variations of the functions $f_{\alpha}^{(k)}(r)$, subject to the same two constraints,

$f_{\alpha}^{(k)}(r) \leqslant 1$;

$$
\begin{aligned}
1 \geqslant & k \equiv N^{-1} \sum_{i j}\left\langle i j\left|\left(1-f^{2}\right)\right| i j-j i\right\rangle \\
& =\frac{\pi \rho}{4 k_{\mathrm{F}}^{2}} \sum_{\alpha, k}(2 T+1)(2 J+1) \frac{1}{2}\left[1-(-1)^{L+S+T}\right] \\
& \times \int_{0}^{\infty} \mathrm{d} r\left[1-f_{\alpha}^{(k)^{2}}(r)\right] a_{\alpha}^{(k)^{2}}\left(k_{\mathrm{F}} r\right),
\end{aligned}
$$

$a_{\alpha}^{(1)^{2}}(x)=x^{2} I_{J}(x)$

and for tensor-correlated channels, $a_{\alpha}^{(2)^{2}}(x)=x^{2}(2 J+1)^{-1}\left\{(J+1) I_{J-1}(x)+J I_{J+1}(x)\right\}$

$a_{\alpha}^{(3)^{2}}(x)=x^{2}(2 J+1)^{-1}\left\{J I_{J-1}(x)+(J+1) I_{J+1}(x)\right\}$,

in terms of the functions $I_{l}(x)$, defined as

$I_{l}(x)=48 \int_{0}^{1} z^{2} \mathrm{~d} z\left(1-\frac{3}{2} z+\frac{1}{2} z^{3}\right) j_{l}^{2}(x z)$.

Imposing the constraint (9) with a Lagrange multiplier $\lambda$, the Euler-Lagrange equations resulting from eq. (7) may be written as

$g_{\alpha}^{(1)^{\prime \prime}}-\left[a_{\alpha}^{(1)^{\prime \prime}} / a_{\alpha}^{(1)}+m \hbar^{-2}\left(V_{\alpha}^{\mathrm{C}}+\lambda\right)\right] g_{\alpha}^{(1)}=0$,

for the tensor-free channels; whereas in each of the tensor-correlated channels we obtain the coupled pair of equations,

$$
\begin{aligned}
& g_{\alpha}^{(2) "}-\left[a_{\alpha}^{(2) "} / a_{\alpha}^{(2)}\right. \\
& \left.+m \hbar^{-2}\left(V_{\alpha}^{\mathrm{C}}+2 V_{\alpha}^{\mathrm{T}}-V_{\alpha}^{\mathrm{LS}}+\lambda\right)+r^{-2} b_{\alpha}^{2} / a_{\alpha}^{(2)^{2}}\right] g_{\alpha}^{(2)} \\
& \quad+\left(r^{-2}-\frac{1}{2} m \hbar^{-2} V_{\alpha}^{\mathrm{LS}}\right) b_{\alpha}^{2}\left\{a_{\alpha}^{(2)} a_{\alpha}^{(3)}\right\}^{-1} g_{\alpha}^{(3)}=0 \\
& g_{\alpha}^{(3)^{\prime \prime}}-\left[a_{\alpha}^{(3)^{\prime \prime}} / a_{\alpha}^{(3)}\right. \\
& \left.\quad+m \hbar^{-2}\left(V_{\alpha}^{\mathrm{C}}-4 V_{\alpha}^{\mathrm{T}}-2 V_{\alpha}^{\mathrm{LS}}+\lambda\right)+r^{-2} b_{\alpha}^{2} / a_{\alpha}^{(3)^{2}}\right] g_{\alpha}^{(3)} \\
& \quad+\left(r^{-2}-\frac{1}{2} m \hbar^{-2} V_{\alpha}^{\mathrm{LS}}\right) b_{\alpha}^{2}\left\{a_{\alpha}^{(2)} a_{\alpha}^{(3)}\right\}^{-1} g_{\alpha}^{(2)}=0
\end{aligned}
$$

where primes denote differentiation with respect to $r$; $a_{\alpha}^{(k)} \equiv a_{\alpha}^{(k)}\left(k_{\mathrm{F}} r\right), b_{\alpha} \equiv b_{\alpha}\left(k_{\mathrm{F}} r\right)$,

where

$b_{\alpha}^{2}(x)=x^{2} 2 J(J+1)(2 J+1)^{-1}\left[I_{J-1}(x)-I_{J+1}(x)\right]$

and as before,

$g_{\alpha}^{(k)}(r)=a_{\alpha}^{(k)}\left(k_{\mathrm{F}} r\right) f_{\alpha}^{(k)}(r)$.

Eqs. (13)-(15) are solved as before to obtain the solutions regular at the origin. The constraints (8) and (9) are imposed by choosing "healing distances" $d_{\alpha}^{(k)}$ as large as possible such that $f_{\alpha}^{(k)}(r)<1$ for $r<d_{\alpha}^{(k)}$, with $f_{\alpha}^{(k)^{\prime}}\left(d_{\alpha}^{(k)}\right)=0$, and $f_{\alpha}^{(k)}(r) \equiv 1$ for $r \geqslant d_{\alpha}^{(k)}$. The Lagrange multiplier $\lambda$ is either set to zero if constraint (9) is satisfied as an inequality, or is varied iteratively, solving the complete set of Euler-Lagrange equations anew at each step, until constraint (9) is satisfied as an equality. 


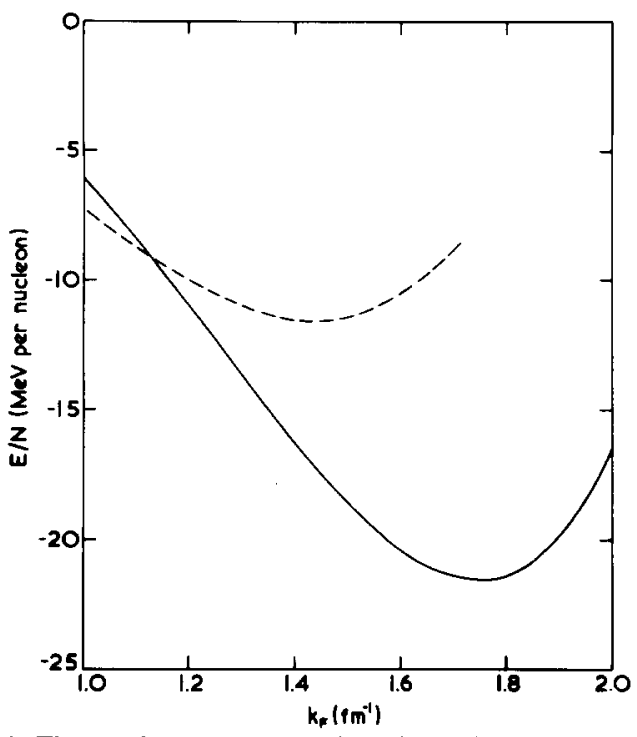

Fig. 1. The total energy per nucleon in nuclear matter using the Reid soft core potential (zero interaction in states $J \geqslant 3$ ). The solid curve and dashed curve give respectively the results of the present calculation and of the LOBT calculation of Siemens and Sprung reported by Bethe [9] .
Performing the calculation outlined above for the Reid soft core interaction [6] assuming no interaction in channels with $J \geqslant 3$, we obtain the saturation curve presented in fig. 1. We see that compared with the LOBT results of Siemens and Sprung presented in the review article of Bethe [9], we obtain considerably greater binding, and saturation at a substantially higher density. Pandharipande [4] has reported LOCV calculations using the Reid potential and an approximate treatment of the tensor force, which agree with the perturbation calculations. More recently $[7,10]$ he has reported the results of many-body calculations which include all of the important many-body clusters (MBC), and quotes a binding energy of $\gtrsim 23 \mathrm{MeV}$ per nucleon at $k_{\mathrm{F}}=1.6 \mathrm{fm}^{-1}$. The discrepancy between the LOBT and MBC calculations is attributed by Pandharipande to dispersion effects [7]. If the NN potential used in these $\mathrm{MBC}$ calculations is indeed equivalent to that used by us, then our variational calculation as presented in fig. 1 is, at least at $k_{\mathrm{F}}=1.6 \mathrm{fm}^{-1}$, in rather better agreement with the MBC results than

Table 1

Contributions to binding at various densities ( $\mathrm{MeV}$ per nucleon). The results of Siemens and Sprung are in parentheses.

\begin{tabular}{|c|c|c|c|c|c|}
\hline$k_{\mathrm{F}}\left(\mathrm{fm}^{-1}\right)$ & 1.0 & 1.2 & 1.4 & 1.6 & 1.7 \\
\hline${ }^{1} \mathrm{~S}_{0}$ & $\begin{array}{c}-7.92 \\
(-8.36)\end{array}$ & $\begin{array}{c}-12.03 \\
(-12.24)\end{array}$ & $\begin{array}{c}-16.62 \\
(-16.35)\end{array}$ & $\begin{array}{c}-21.36 \\
(-20.30)\end{array}$ & $\begin{array}{c}-23.65 \\
(-22.10)\end{array}$ \\
\hline${ }^{1} \mathrm{P}_{1}$ & $\begin{array}{c}0.45 \\
(0.55)\end{array}$ & $\begin{array}{c}0.98 \\
(1.30)\end{array}$ & $\begin{array}{c}2.03 \\
(2.76)\end{array}$ & $\begin{array}{c}3.92 \\
(5.34)\end{array}$ & $\begin{array}{c}5.31 \\
(7.17)\end{array}$ \\
\hline${ }^{1} \mathrm{D}_{2}$ & $\begin{array}{c}-0.65 \\
(-0.65)\end{array}$ & $\begin{array}{c}-1.47 \\
(-1.48)\end{array}$ & $\begin{array}{c}-2.87 \\
(-2.89)\end{array}$ & $\begin{array}{c}-5.04 \\
(-5.08)\end{array}$ & $\begin{array}{c}-6.47 \\
(-6.54)\end{array}$ \\
\hline${ }^{3} \mathrm{P}_{0}$ & $\begin{array}{c}-1.34 \\
(-1.34)\end{array}$ & $\begin{array}{c}-2.40 \\
(-2.36)\end{array}$ & $\begin{array}{c}-3.70 \\
(-3.56)\end{array}$ & $\begin{array}{c}-5.09 \\
(-4.77)\end{array}$ & $\begin{array}{c}-5.75 \\
(-5.22)\end{array}$ \\
\hline${ }^{3} \mathrm{P}_{1}$ & $\begin{array}{c}2.69 \\
(3.02)\end{array}$ & $\begin{array}{c}5.50 \\
(6.15)\end{array}$ & $\begin{array}{c}10.00 \\
(11.08)\end{array}$ & $\begin{array}{c}16.78 \\
(18.30)\end{array}$ & $\begin{array}{r}21.24 \\
(22.90\end{array}$ \\
\hline${ }^{3} S_{1}$ & $\begin{array}{c}-9.07 \\
(-10.15)\end{array}$ & $\begin{array}{l}-13.60 \\
(-13.08)\end{array}$ & $\begin{array}{c}-18.21 \\
(-15.19)\end{array}$ & $\begin{array}{c}-22.20 \\
(-15.89)\end{array}$ & $\begin{array}{c}-23.68 \\
(-15.67)\end{array}$ \\
\hline${ }^{3} \mathrm{D}_{1}$ & $\begin{array}{c}0.28 \\
(0.40)\end{array}$ & $\begin{array}{c}0.65 \\
(0.87)\end{array}$ & $\begin{array}{c}1.30 \\
(1.62)\end{array}$ & $\begin{array}{c}2.27 \\
(2.67)\end{array}$ & $\begin{array}{r}2.88 \\
(3.31)\end{array}$ \\
\hline${ }^{3} \mathrm{D}_{2}$ & $\begin{array}{c}-1.14 \\
(-1.17)\end{array}$ & $\begin{array}{c}-2.54 \\
(-2.58)\end{array}$ & $\begin{array}{c}-4.79 \\
(-4.85)\end{array}$ & $\begin{array}{c}-8.05 \\
(-8.13)\end{array}$ & $\begin{array}{c}-10.08 \\
(-10.19)\end{array}$ \\
\hline $\left.\begin{array}{c}{ }^{3} \mathrm{P}_{2} \\
{ }^{3} \mathrm{~F}_{2}\end{array}\right\}$ & $\begin{array}{c}-1.67 \\
(-1.93) \\
-0.11 \\
(-0.11)\end{array}$ & $\begin{array}{c}-3.73 \\
(-4.21) \\
-0.30 \\
(-0.30)\end{array}$ & $\begin{array}{c}-7.17 \\
(-7.91) \\
-0.64 \\
(-0.64)\end{array}$ & $\begin{array}{c}-12.29 \\
(-13.36) \\
-1.19 \\
(-1.20)\end{array}$ & $\begin{array}{c}-15.56 \\
(-16.84) \\
-1.56 \\
(-1.58)\end{array}$ \\
\hline Sum, $E_{2}$ & $\begin{array}{c}-18.47 \\
(-19.74)\end{array}$ & $\begin{array}{c}-28.93 \\
(-27.93)\end{array}$ & $\begin{array}{c}-40.67 \\
(-35.91)\end{array}$ & $\begin{array}{c}-52.26 \\
(-42.40)\end{array}$ & $\begin{array}{c}-57.34 \\
(-44.76)\end{array}$ \\
\hline Kinetic, $E_{1}$ & 12.44 & 17.92 & 24.38 & 31.85 & 35.95 \\
\hline Binding, $-\left(E_{1}+E_{2}\right)$ & $\begin{array}{r}6.03 \\
(7.30\end{array}$ & $\begin{array}{c}11.02 \\
(10.01)\end{array}$ & $\begin{array}{c}16.28 \\
(11.53)\end{array}$ & $\begin{array}{c}20.41 \\
(10.55)\end{array}$ & $\begin{array}{l}21.38 \\
(8.81)\end{array}$ \\
\hline
\end{tabular}


with the results of other lowest order calculations which suggests that the discrepancy is perhaps attributable to the inadequacy of the LOCV prescription of Pandharipande in particular rather than to an inherent feature of low order variational calculations in general. Indeed, if one views the MBC calculations as a good approximation to the exact solution of the problem then we have the result that our variational approach yields excellent agreement for the "homework problem" of Bethe and now encouraging agreement with the nuclear matter "exact" solutions.

It is interesting to investigate the origin of the discrepancy between our variational results and the results of perturbation calculations. In table 1 we compare our results with those of Sprung and Siemens reported by Bethe [9] channel by channel. We note that there is substantial agreement between the two calculations in all channels except the ${ }^{3} \mathrm{~S}_{1}-{ }^{3} \mathrm{D}_{1}$ channel. At high densities the discrepancy in the ${ }^{3} \mathrm{~S}_{1}-{ }^{3} \mathrm{D}_{1}$ channel is sufficient to account for almost all the discrepancy in the total binding energy, and it is this discrepancy that Pandharipande [7] has attributed to dispersion effects. Whether this is the case or whether the discrepancy may not be due to strong tensor correlations which are poorly accounted for in low order perturbation calculations is not clear to us. This point remains to be more fully investigated.

It has become the practice to select models of the
NN interaction for use in nuclear physics calculations by their ability to fit simultaneously the two-body NN data and the saturation properties of nuclear matter. If our variational results can be trusted, they would suggest that LOBT calculations might not be an adequate test of the saturation properties of an interaction, and they would also cast doubt on the suitability of the Reid interaction as a representation of the NN force. It is clear that these points will only be fully resolved when complete $\mathrm{MBC}$ calculations for the Reid potential become available.

\section{References}

[1] J.C. Owen, R.F. Bishop and J.M. Irvine, Phys. Lett. B, to be published.

[2] J.C. Owen, R.F. Bishop and J.M. Irvine, submitted to Ann. Phys. (NY).

[3] V.R. Pandharipande, Nucl. Phys. A174 (1971) 641.

[4] V.R. Pandharipande, Nucl. Phys. A1 81 (1972) 33.

[5] L. Shen and C.-W. Woo, Phys. Rev. D10 (1974) 371.

[6] R.V. Reid, Ann. Phys. (NY) 50 (1968) 411.

[7] V.R. Pandharipande, R.B. Wiringa and B.D. Day, Phys. Lett. 57B (1975) 205.

[8] M.L. Ristig, W.J. Ter Louw and J.W. Clark, Phys. Rev. C3 (1971) 1504.

[9] H.A. Bethe, Ann. Rev. Nucl. Sci. 21 (1971) 93.

[10] V.R. Pandharipande and R.B. Wiringa, to be published. 